\title{
La secuencia arqueológica en el cinturón forestal centroafricano
}

\author{
Raquel Martí *
}

\begin{abstract}
RESUMEN
Este trabajo presenta una revisión de la evidencia arqueológica en el cinturón forestal de Africa Central desde el Pleistoceno Final al Holoceno reciente. El avance generado por la investigación en Africa en las últimas décadas ha mostrado una mayor complejidad y variabiiidad en el patrón de cambio tecnológico de lo que en un principio se estableció. Estos patrones

además, difieren en gran medida de los observados en el registro europeo.
\end{abstract}

ABSTRACT

This paper explores from the Late Pleistocene to the Late Holocene archaeological record of Central Africa. The advance provided in Africa by the research during the last decades has shown a more complexity and variability in the technological change pattern than earlier believed. These patterns are quite different from the European record.

KEY WORDS

Rainforest, Central Africa, Late Pleistocene, Holocene. Archaeology, Paleoenvironment.

\section{INTRODUCCIÓN}

La información disponible en los bosques lluviosos tropicales sobre el origen de la ocupación humana, así como de sus estrategias de subsistencia a

* Becaria Predoctoral del Dpto. de Prehistoria de la UNED; C/ Senda del Rey s/n; 28047 Madrid.rmlezana@eresmas.com. 
lo largo del pasado, es reducida. Este vacío de información se debe principalmente al escaso desarrollo alcanzado por la investigación en estos contextos. Sólo en las últimas décadas, la creación de hipótesis relacionadas con la antigüedad y origen de su poblamiento, así como las relativas a los cambios paleoambientales han impulsado el avance de la investigación.

El interés suscitado en los últimos tiempos por el estudio de los bosques lluviosos tropicales radica en que a través de ellos se adquiere un mayor conocimiento sobre la capacidad de la especie humana en la colonización de diferentes ecosistemas, así como sobre su flexibilidad en la adaptación a los diferentes cambios medioambientales acaecidos durante el pasado. Además de ello, su estudio desvela cómo la población humana ha intervenido en el desarrollo de uno de los ecosistemas más complejos de la Tierra.

En la actualidad se estima una ocupación de los bosques húmedos tropicales del globo al menos desde el Pleistoceno final. En el cinturón forestal centroafricano, la población humana estaba establecida alrededor del $40.000 \mathrm{BP}$. A pesar de la existencia de hallazgos mucho más antiguos, la falta de datos paleoambientales asociados a esta evidencia impide el conocimiento sobre el tipo de contexto medioambiental en el que se desarrollaron.

El bosque lluvioso tropical tal y como lo observamos hoy en día se estableció, en parte, hace aproximadamente 12.000 años. Con anterioridad a esta fecha, las fluctuaciones medioambientales han influido en la composición del paisaje forestal y en consecuencia, los patrones de ocupación de Africa Central y las estrategias de subsistencia han debido de variar a lo largo del tiempo.

\section{LA REGIÓN DE ESTUDIO}

La vegetación de los bosques de Africa Central pertenece al dominio "Guineo-Congolés», el cual comprende diversos tipos de bosque denso húmedo de tierras bajas según las características de las especies perennes o semicaducifolias que lo componen (White, 1983). Existen además numerosas formaciones transicionales difícilmente clasificables entre los bosques típicamente semicaducifolios o perennes.

Estas formaciones forestales se desarrollan bajo unas condiciones determinadas relacionadas con su localización latitudinal tropical (temperatura, humedad y pluviometría elevada) y su posición altitudinal: la máxima elevación sobre la que se desarrollan es de 1.000 metros, es por ello que 
se les define como bosques de tierras bajas. A partir de los 1.000 metros, las formaciones son transicionales hacia estructuras y fisionomías típicas de bosque afromontano.

El cinturón forestal africano se extiende desde la costa atlántica, por toda la Cuenca del Congo hasta la dorsal del Kivu en la región interlacustre del Rift africano (Fig. 1). La prolongación de este bosque forestal centroafricano se produce desde Guinea a Ghana, quedando ambos separados por el vacío de Dahomey o Benin.

La Cuenca del Congo presenta una altitud media de 400 metros, aumentando gradualmente hacia las tierras altas y plateaux que forman su límite. En la Cuenca, las rocas Precámbricas están cubiertas por sedimentos continentales depositados desde el Palaeozoico a la actualidad. Los sedimentos cuaternarios cubren 150.000 kilómetros cuadrados de planicies aluviales en el centro de la Cuenca, donde se localizan todavía formaciones lacustres y extensas áreas pantanosas. En los alrededores de la Cuenca del Zaire se sitúan las tierras altas ecuatoriales, una región de plateaux diseccionados, compuestos en su mayoría por rocas ligeramente metamorfizadas del Precámbrico Superior. Hacia el este de la Cuenca las alturas aumentan en dirección a las colinas del Kivu. Estas colinas consisten principalmente en rocas Precámbricas fuertemente metamorfizadas. Las colinas más altas, fuera ya del dominio GuineoCongolés, están cubiertas de vegetación afromontana. Hacia el Noroeste, el plateau ondulado del Este de Camerún presenta una elevación de 600 a 800 metros, alcanzando los 1.500 metros de altura o más en las tierras altas de Bamenda-Adamawa. Al oeste del borde de la Cuenca de Zaire, el contacto con la estrecha planicie costera atlántica

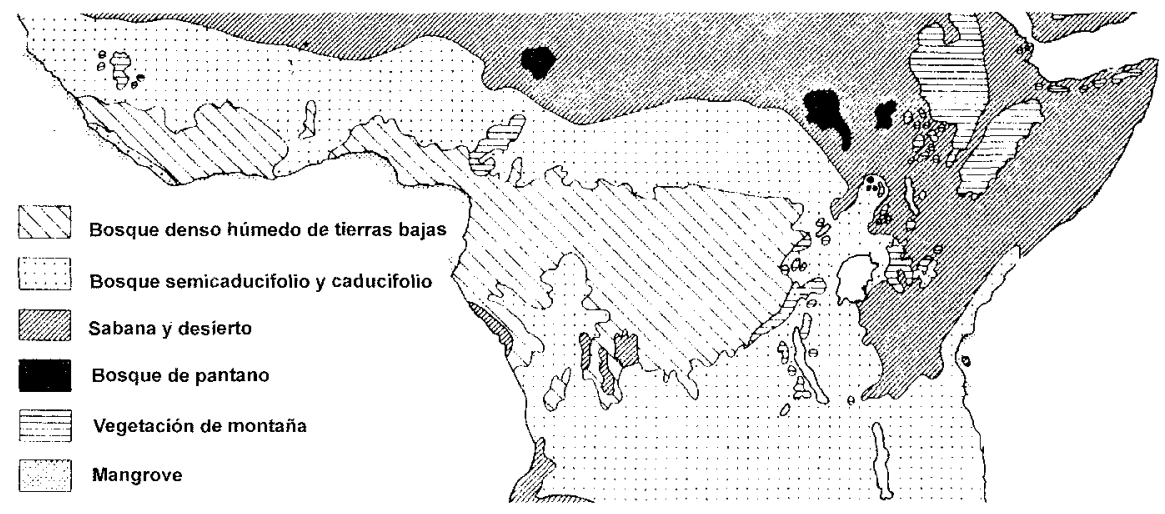

Fig. 1. Mapa de vegetación de Africa Ecuatorial (Flenley, 1979). 
esta formada por los plateaux de Camerún-Gabón con altitudes entre 600 y 1.000 metros, los cuales se extienden hacia el sur (Montes de Cristal y Montes del Mayombe) y están formados por rocas metamórficas. Por último, la planicie costera, entre Angola y Camerún, presenta diferente anchura, aumentando sobre todo cerca de Libreville y en los alrededores de Douala. La planicie está cruzada por numerosos ríos con formaciones de manglares que bordean los estuarios. En esta zona se localizan también lagunas, lagos y pantanos.

Las características geológicas y los principales rasgos geomorfológicos quedan detallados en la Fig. 2.

\section{LA SECUENCIA PALEOAMBIENTAL}

La configuración de la vegetación en los bosques tropicales de Africa ha variado a lo largo del tiempo. A la hora de abordar el estudio del asentamiento humano en los bosques tropicales es necesario tener en cuenta tales variaciones puesto que han debido influir en la colonización, asentamiento y estrategias de explotación de las poblaciones a lo largo del pasado.

El bosque observable en la actualidad en Africa Central se estableció, en parte, desde el comienzo del Holoceno, hace 12.000 años. Durante el Pleistoceno las fluctuaciones climáticas (glaciales: episodios fríos y secos; interglaciales: episodios cálidos y húmedos), ocasionaron cambios en el paisaje forestal, sin embargo, la extensión, distribución y estructura de la vegetación durante este periodo no se conoce con exactitud. Durante los periodos glaciales, parece ser que en algunas regiones las formaciones forestales dieron paso a paisajes áridos y abiertos, mientras que durante los interglaciales se produjo la recuperación de la masa forestal.

La secuencia paleoambiental del Pleistoceno Antiguo, Medio y parte del Superior es prácticamente desconocida. La mayor parte de la información paleoecológica disponible se encuadra en la última glaciación (70.000 $\mathrm{BP}-10.000 \mathrm{BP}$ ) o en el Holoceno. Las grandes fases climáticas en las que se ha dividido son:

- Maluekiense (70.000 BP-40.000 BP): descenso térmico, aridez y deforestación.

- Njiliense (40.000 BP-30.000 BP): fase interestadial cálida y húmeda, en la que se produce una reforestación.

- Leopoldvilliense (30.000 BP-12.000 BP): último estadial. Enfriamiento y tendencia a la sequía que provoca el descenso del cinturón latitudinal de 

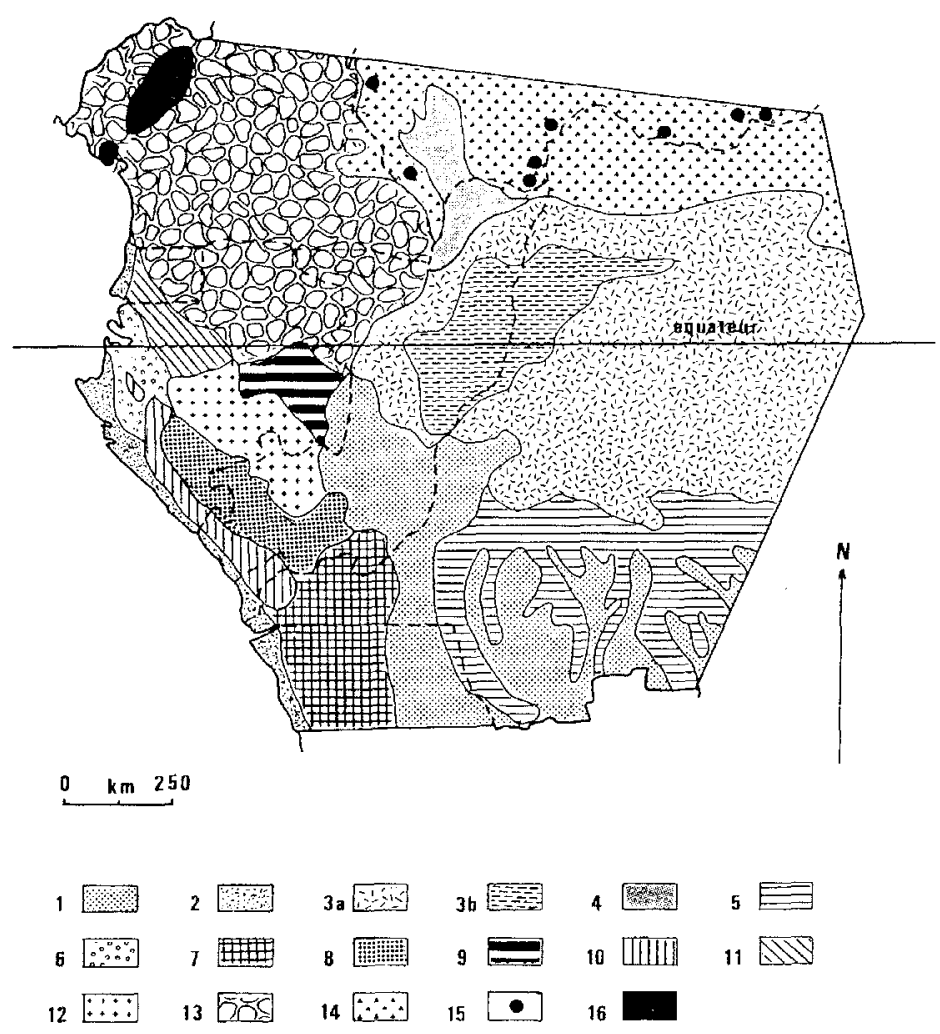

Fig. 2. Mapa Geológico y rasgos geomorfológicos de África Central (Schwartz y Lanfranchi, 1993).

1. Arenas ocres neógenas del Plateau y colinas de Bateke. 2. Arenas Plio-Pleistocenas de la serie de "Cirques" de la planicie costera y sus equivalentes. 3. Plioceno y Pleistoceno de la cubeta congolesa: $3 a$ zona externa, paisajes de mesetas onduladas; $3 b$ zona interna, depresión. 4.Gres mesozoico de Carnot, Plateau de Gadzi. 5. Valles aluviales del sur de la cubeta congolesa (formaciones cretácias recubiertas de aluviones cuaternarios). 6. Areniscas cretáceas, argilitas y margas de la cuenca sedimentaria costera gabonesa (Cretáceo), alternancia de colinas y de zonas depresivas hidromorfas. 7. Gres rojo de Inkisi (Precámbrico superior) formando el Plateau de Cataractes y su prolongación hacia el sur. 8. Synclinorium de Niari-Nyanga. Esquistos calcáreos y gres precámbricos. Paisaje de colinas. 9. Cubeta sedimentaria (Precámbrico medio) de Franceville, con paisajes muy variados. 10. Mayombe, Cadena montañosa formada de rocas metamórficas precámbricas (esquistos y cuarcitas). Paisaje de cadenas paralelas y valles. 11. Montes de Cristal gabonés. Migmatitas y granitos del Precámbrico inferior. Región de colinas muy accidentada. 12. Batolitos graniticos de Chaillu. Región de montañas medianas que incluyen colinas $y$ zonas tabulares. 13. Rocas metamórficas y magmatitas precámbricas de plateaux, disecciones en colinas, norte gabonés y sur camerunés. 14. Dorsal centroafricana (Precámbrico cristalofiliense y rocas cristalinas indiferenciadas). 15. Zonas particularmente ricas en inselbergs y en afloramientos ferruginosos. 16. Zona volcánica de la dorsal camerunesa. 
vegetación de montaña a las Tierras Bajas. Posterior aridez extrema con predominio de espacios abiertos y ausencia de vegetación en torno al cénit hipotermal del 18.000 BP. En el 14.500 BP se produce el restablecimiento de la vegetación arbórea semejante a la existente al inicio de esta fase y alrededor del 12.500 BP tendrá lugar la expansión del bosque denso húmedo de Tierras Bajas (Adebisi, 1991).

- Kibanguiense (12.000 BP a la actualidad):

- Kibanguiense A (12.000 BP-4.000 BP): mayor humedad y elevación térmica. Expansión forestal más allá de sus límites actuales.

- Kibanguiense B (4.000 BP-actualidad): apertura y/o mutabilidad del bosque debido a episodios de sequía, incendios naturales e instrumentales y otras perturbaciones ambientales (Hart, et al; 1996) en dos subfases: 4.000 BP-3.000 BP (Schwartz, 1992) y 2.500 BP-2.000 BP (Maley, 1992).

Una de las principales impulsoras en la investigación de la paleoecología tropical en las últimas décadas fue la denominada hipótesis de los "refugios forestales». Esta hipótesis fue presentada primeramente por Haffer en 1969 en los Neotrópicos y posteriormente por Hamilton en 1972-74 en Africa. Partiendo de la observación actual de la distribución biogeográfica de especies, estos autores buscaron una interpretación que explicara la distribución y alto grado de endemismo de pájaros, primates, mariposas y ciertas especies leñosas, en diferentes puntos aislados de la Cuenca del Congo, frente a otras zonas en las que la hibridación y escaso endemismo era la característica dominante. Para explicar este alto grado de endemismo, era necesario que estas especies hubiesen sufrido un aislamiento prolongado y recurrieron a un criterio paleoclimático como mejor candidato causal de tal aislamiento. Así, las oscilaciones glaciales del Pleistoceno produjeron una bajada de las temperaturas, una disminución de la humedad con la consecuente fragmentación de la masa forestal y por último, la creación de conjuntos insulares boscosos rodeados de sabana.

Estas zonas de gran endemismo hoy día en Africa Central, fueron hipotéticamente relacionadas con la existencia de antiguas islas boscosas (refugios forestales) durante las condiciones hipotermales del Pleistoceno. El aislamiento prolongado en estas islas rodeadas de sabana, llevó a la creación de áreas de gran especiación y endemismo tanto floral como faunístico. Posteriormente, el mejoramiento climático de las fases interglaciales, impulsó la expansión forestal a partir de estos refugios o áreas nucleares sobre las zonas de sabanas, poniendo fin a tal aislamiento y creando así, zonas de escaso endemismo y gran hibridación. A. Hamilton (1972-74) 
situó estos refugios en el sur de Camerún/Gabón, en el bloque Guineano y al oeste de Ruwenzori / Kivu (República Democrática del Congo) (Fig. 3).

Esta hipótesis fue rápidamente aceptada en el ámbito académico y todas estas zonas pasaron a ser consideradas "refugios forestales" sin ningún tipo de validación. Sin embargo, la evidencia paleoambiental presentada en los últimos años en las zonas consideradas como refugios forestales (Adebisi, 1991, Hart, et al, 1996; Runge, 1996; Mercader, et al, (e.p.), no permite mantener la existencia de masas forestales estáticas durante el pasado, sino que por el contrario se registra procesos de cambios dramáticos en la estructura, composición y distribución de la vegetación a través del tiempo.

Por otra parte, como ya sugería J. Mercader (1997), varios investigadores han demostrado que no toda la especiación documentada hoy día tuvo su origen en estos refugios forestales, sino que diferentes circunstancias ambientales como la pluviometría, los suelos, la existencia de grandes barreras fluviales, la aparición de ciertos eventos geomorfológicos de gran magnitud (Gentry, 1989; Van der Hamment, 1991) y las alteraciones hidráulicas por inundación (Colinvaux, et al, 1985), justifican parte de la especiación. Además, es altamente improbable, que todos los endemismos se hayan desarrollado durante los últimos 10.000 años, así como que todos los endemismos de una localidad restringida, hayan emigrado

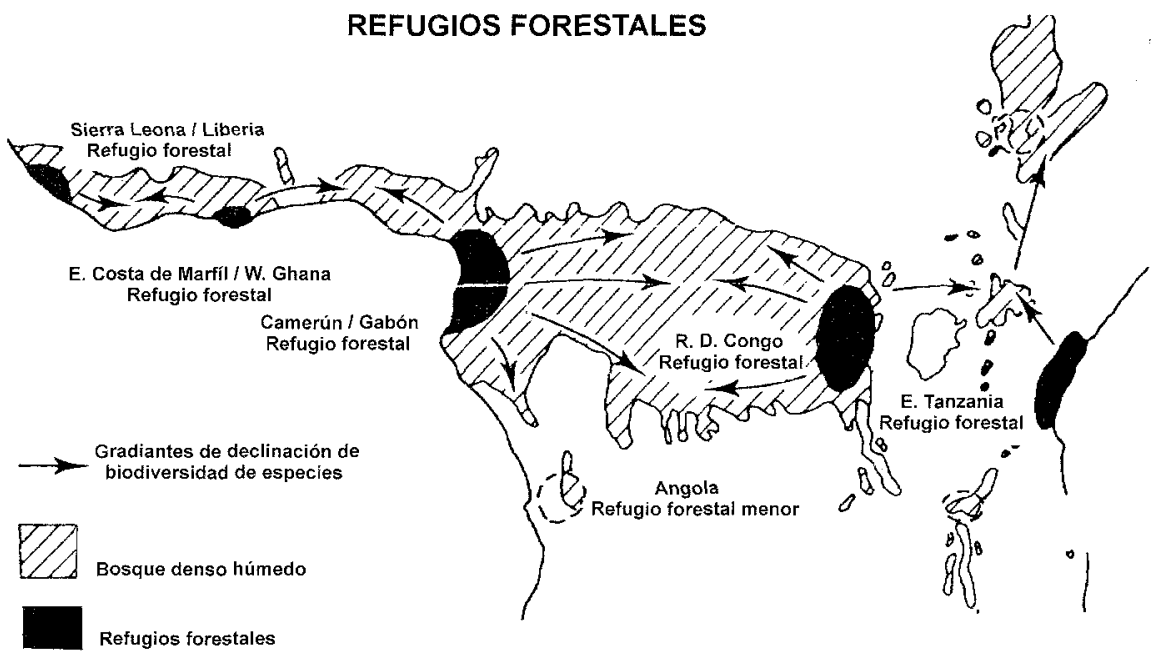

Fig. 3. Refugios forestales en Africa Ecuatorial (Hamilton, 1972-74). 
desde un refugio forestal distante, en el mismo espacio de tiempo (Livingstone, 1980).

Estos datos sugieren una evolución paleoambiental mucho más compleja de lo que la hipótesis de los refugios forestales sugería. Las pluvisilvas son dinámicas y variables a escala temporal, por lo que no es probable que existiese una masa forestal única que se fragmentó, redujo y creo reductos, que según el modelo de los refugios forestales gozaría de la misma estabilidad y la misma composición forestal que durante los periodos de máxima humedad. Sin lugar a dudas, a lo largo de las fases áridas algunas áreas favorecidas por su posición topográfica han mantenido una masa forestal, mientras que otras zonas probablemente se habrán caracterizado por la presencia de espacios abiertos. La persistencia de la vegetación en ciertos lugares no significa que la estructura y composición haya sido la misma durante las diferentes fases climáticas, sino que por el contrario la masa forestal ha soportado transformaciones notables a lo largo del tiempo.

\section{EL DESARROLLO DE LA INVESTIGACIÓN ARQUEOLÓGICA EN AFRICA CENTRAL}

Las características socio-políticas de los países centroafricanos, así como los problemas logísticos implícitos en este tipo de ecosistemas limitan el desarrollo de la investigación arqueológica. Por otra parte, el diseño de las investigaciones llevadas a cabo en Africa Central rara vez incluyen información paleoambiental, su enfoque dominante es tecno-tipológico, presentando pocas veces información sobre los procesos de formación del registro arqueológico y estando la mayor parte de las veces centrada en los periodos más recientes en detrimento de los más antiguos. Por último el número de yacimientos excavados y de investigadores trabajando a lo largo de todo el cinturón forestal es reducido: se estima que únicamente una veintena de arqueólogos han trabajado durante los últimos veinte años en un territorio diez veces más grande que Francia (Maret, 1984).

Es debido a todos estos factores por lo que la investigación arqueológica en Africa Central se encuentre en sus inicios. Partiendo de la base de que es prácticamente imposible establecer una secuencia a escala local, dado el estado actual del conocimiento, menor es la posibilidad de hablar a escala regional. A pesar de todos ello, su estudio genera la construcción de hipótesis que son la base fundamental a partir de la cual se desarrolla toda investigación y, es debido a esta juventud de la arqueología centroafricana que cada vez que se genera más investigación, las antiguas hipótesis se tambalean. 
Dos grandes hipótesis han girado en torno a la interpretación de la ocupación humana en el bosque tropical. Una de las más extendidas en el estudio de Africa Central es la hipótesís de la migración bantú (Oliver, 1966; Bouquiaux, 1977; Ehret, 1984; Vansina, 1984; 1985) que ha servido para interpretar el origen y significado de la mayor parte de los yacimientos del Holoceno reciente en Africa Central.

En la actualidad un total de 450 lenguas bantúes son habladas por ciento cincuenta millones de individuos que habitan al sur de una línea situada desde Nigeria a Kenya. Esta distribución homogénea de lenguas bantúes es, según los lingüistas, resultado de un fenómeno de difusión o de migración de población a partir de un centro único, situado entre la frontera de Nigeria y Camerún, en las Grassfields. Los estudios de glotocronología indican que los Proto-bantúes eran agricultores, fabricaban cerámica y poseían el conocimiento de la fundición de metales. Es a partir de estos movimientos de población, según los autores arriba mencionados, cuando las tecnologías cerámicas y metalúrgicas, así como las prácticas agrícolas se difunden por Africa desde los centros bantúes originarios.

La segunda hipótesis, quizá menos conocida fuera del ámbito de los estudios del bosque tropical, es la llamada «hipótesis nula». Esta hipótesis plantea que con anterioridad a la colonización agrícola en estos ecosistemas, no existieron poblaciones capaces de sobrevivir en los bosques tropicales del globo. Esta premisa se basó en la interdependencia observada en el marco etnográfico entre cazadores-recolectores y agricultores, según la cual, los cazadores-recolectores obtienen en la actualidad más de la mitad de su dieta, basada en carbohidratos, mediante el intercambio con los agricultores. Esta observación llevó a algunos autores a la hipótesis de que la ocupación humana en las pluvisilvas no fue posible hasta la aparición de la agricultura en estos contextos (Hart y Hart, 1986; Bailey et al, 1989).

Esta hipótesis muy de moda en los últimos años, generó el inicio de una amplia investigación a escala mundial en busca de las evidencias que demostraran su valía e impulsó ampliamente los estudios arqueológicos en los bosques tropicales. La contestación a esta hipótesis no se hizo esperar y fueron varios autores (Colinvaux y Bush, 1991; Bahuchet, et al, 1991) los que criticaron el actualismo de base de la "hipótesis nula». Finalmente, la evidencia arqueológica aportada en los últimos años en varias regiones del mundo (Sureste Asia / Sahul: ej. Bellwood, 1985; Horsfall, 1987; Neotrópicos: ej. Ranere, 1972; Roosevelt, et al, 1966; Africa: ej. Coon, et al, 1968; Mercader, 1997), demostró la existencia de ocupaciones prehistóri- 
cas con economías cazadoras-recolectoras en estos contextos con anterioridad a la aparición de poblaciones productoras. Los resultados de esta investigación proporcionaron una sólida base que permitió refutar la «hipótesis nula» y concluyeron con la consideración de que los Bosques Lluviosos Tropicales fueron colonizados desde época pre-agrícola.

\section{LA SECUENCIA ARQUEOLÓGICA EN AFRICA CENTRAL}

Durante la celebración del Tercer Congreso Panafricano en 1955, se estableció una división general de los periodos más antiguos de la Prehistoria en el continente africano: Early Stone Age (ESA), Middle Stone Age (MSA) y Late Stone Age (LSA), un esquema que en rasgos generales coincidía con el Paleolítico Inferior, Medio y Superior europeo (Clark and Cole, 1957). Posteriormente durante la conferencia de Burg Wartenstein en 1965 se hizo patente la necesidad de abandonar esta división de la Prehistoria (Bishop y Clark, 1967), ante la realidad de una evidencia arqueológica mucho más compleja. A pesar de este intento muchos autores continúan utilizando esta división de las antiguas Edades de la Piedra, debido a que no se ha incorporado ninguna alternativa a la nomenclatura formal. En el caso concreto de la región centroafricana el registro arqueológico presenta también características propias que no permite aplicar una secuencia rígida de patrones de cambio tecnológico.

Los datos más antiguos han sido identificados como Early Stone Age. La mayor parte de los hallazgos atribuidos a este complejo se encuentra en depósitos alterados, desmantelados o en superficie, cuya asignación se ha basado principalmente en la presencia de cantos trabajados $\mathrm{y} / \mathrm{o}$ bifaces. La evidencia atribuida a estos complejos es fragmentaria, sus hallazgos se concentran principalmente en la región del Alto Sangha de la República Centroafricana (Bayle des Hermens, 1975). Las industrias (Fig. 4), localizadas en contextos fluviales, se presentan a veces muy rodadas, casi todas fuertemente patinadas y en otros casos mezcladas con industrias más recientes. La República Democrática del Congo, por el contrario, presenta los dos únicos yacimientos Achelenses, Kamoa (Cahen, 1982; Cahen y Moeyersons, 1969-71) y Pointe de la Gombe (Cahen, 1976, 1982) con información contextual de todo Africa Central. En el primero, el conjunto industrial esta formado por bifaces, hendedores, puntas triédricas, picos y numerosas raederas, todas ellas sobre lasca. La talla levallois, kombewa y victoria han sido también identificados en el conjunto. El yacimiento de Pointe de la Gombe presenta además, una de las secuencias más completas de Africa Central, desde un nivel 

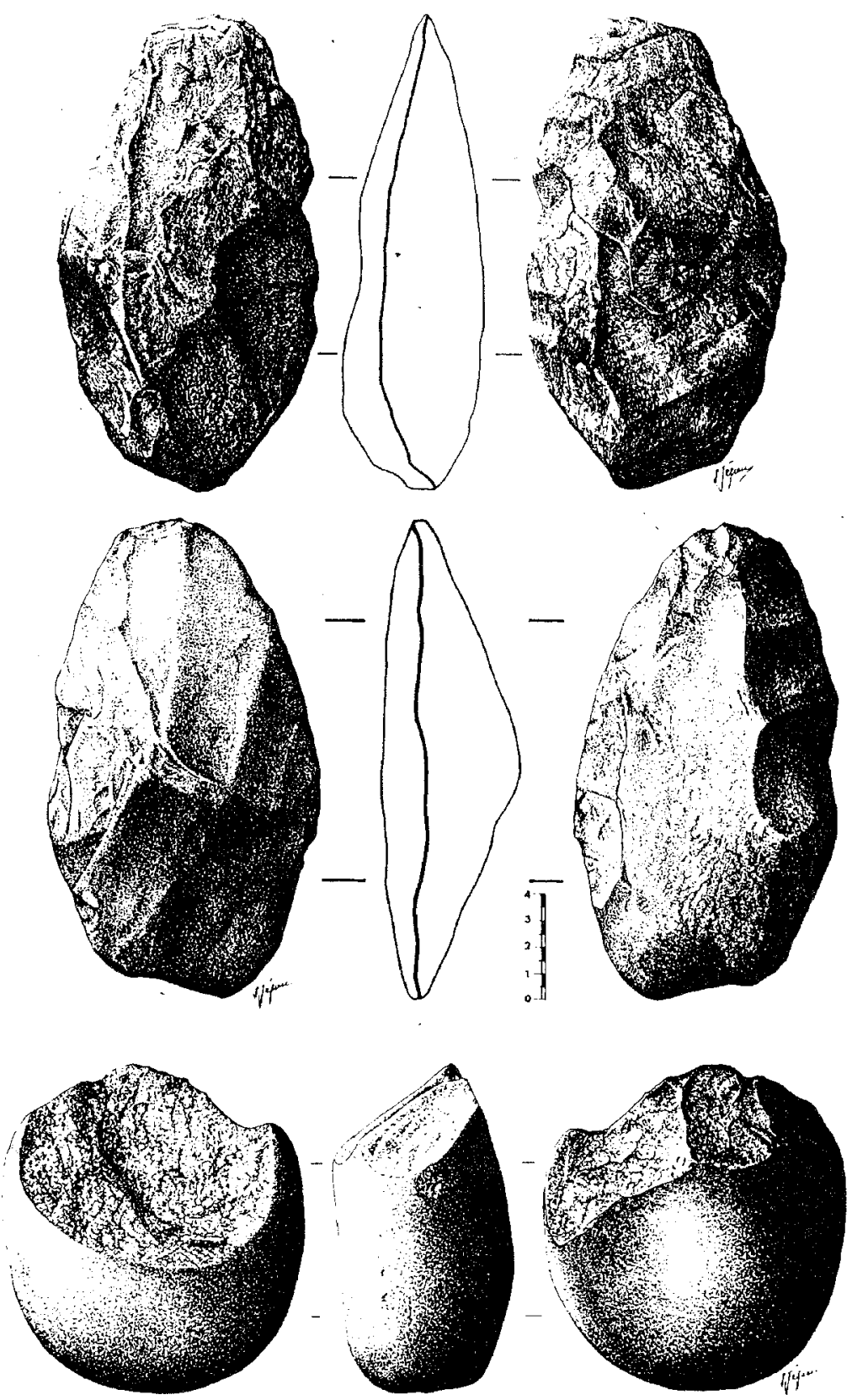

Fig. 4. Industria lítica de la ESA de la República Centroafricana (Bayle des Hermens, 1975). 
basal de gravas con industrias sobre lasca Achelenses, hasta niveles del Holoceno reciente.

Los datos disponibles para este tecno-complejo no presentan casi ninguna información cronológica, socioeconómica o paleoambiental (con la excepción de la identificación de especies de sabana en Kamoa en el momento de la ocupación del enclave), reduciéndose en la mayoría de los casos a meros datos tipológicos, sin embargo, su existencia al menos indica una presencia humana temprana en Africa Central.

En un momento posterior se desarrollan los complejos Sangoenses y Lupembienses, comprendiendo aquellas industrias que enlazan las últimas manifestaciones achelenses todavía documentables en contextos sangoenses y los primeros artefactos del Pleistoceno Final y Holoceno Inicial. Ambas, además se solapan entre sí. Tanto Sangoense como Lupembiense son dos periodos a todas luces mal conocidos. Su cronología, datos paleoambientales, composición de sus industrias y procesos de formación de los yacimientos que las contienen son conocidos de una forma muy fragmentaria.

Convencionalmente, se les engloba en un periodo comprendido entre el $70.000 \mathrm{BP}$ y el $12.000 \mathrm{BP}$, el cual incluye tres grandes fases climáticas con diferentes periodos áridos y secos (Malukiense y Leopoldvilliense), así como cálido y húmedo (Njiliense). En el resto de Africa Subsahariana, la edad del Sangoense podría remontarse al menos a los últimos 100.000 años (McBrearty, 1987).

La cronología en Africa Central para estas industrias es prácticamente desconocida: en Lunda, Angola, al sur de la Cuenca del Congo, el Sangoense-Lupembiense inferior ha sido datado en 38.000 BP (UCLA168) (Clark, 1963); en la Planicie de Kinshasa (República Democrática del Congo), el Lupembiense presenta cronologías de $>30.000$ BP (Lv.- 44/47) $y>26.000$ BP (Lv.-163) (Van Moorsel, 1968), mientras que en el yacimiento de Pointe de la Gombe (República Democrática del Congo) un nivel con artefactos sangoenses-lupembienses presenta una datación más controvertida que supera los $43.800 \mathrm{BP}$ (GrN-7277) junto con otra mucho más reciente en el mismo nivel de $27.240 \pm 280 \mathrm{BP}(\mathrm{GrN}-7221)$ (Cahen, 1976). Otras fechas más tardías sitúan al Lupembiense en los últimos milenios del Pleistoceno superior: 15.080 BP \pm 480 (Lv-166) y $12.230 \pm 250$ BP (Lv-287) en la Planicie de Kinshasa (Van Moorsel, 1968); $14.503 \pm$ $560 \mathrm{BP}(\mathrm{C}-580) ; 11.189 \pm 490 \mathrm{BP}(\mathrm{C}-580)$ en Lunda (Clark, 1963) y 14.840 $\pm 80 \mathrm{BP}$ (GrN 7276) en Pointe de la Gombe (Cahen; 1976).

Las industrias sangoenses se caracterizan por la presencia de utillaje macrolítico (cantos trabajados, picos y bifaces), aunque en algunos yaci- 
mientos los útiles de menores dimensiones como los raspadores, son los elementos dominantes en el conjunto. El Sangoense fue definido como un conjunto industrial adaptado a un medio forestal, donde el repertorio artefactual estaba destinado al trabajo de la madera (Clark, 1964; 1982; Davies, 1967; Cahen, 1978), sin embargo, tanto el Sangoense como el Lupembiense son industrias que se desarrollan durante las fases climáticas áridas, frías y de paisajes abiertos que desde el 70.000 BP hasta el $12.000 \mathrm{BP}$, sólo incluyen una fase forestal: el interestadial Njiliense (40.000-30.000 BP).

El Lupembiense, se caracteriza por presentar en sus conjuntos, elementos más sofisticados (puntas bifaciales foliáceas trabajas mediante finos retoques por presión) (Fig. 5), junto con la pervivencia de elementos más arcaicos (cantos trabajados y picos) en una fase inicial. Posteriormente se inicia una tendencia de disminución del tamaño de los materiales, así como una diversificación de las puntas (Van Moorsel, 1968). Enlazando con los conjuntos del Pleistoceno Final y Holoceno, continúa la tendencia a la reducción de las dimensiones de los artefactos e incluye hojas, hojitas y las primeras puntas de flecha registradas en estos contextos.

Estos conjuntos industriales se encuentran depositados, en la mayoría de los casos, en formaciones pedológicas de líneas de piedras, «Stoneline», así como en los sedimentos areno-arcillosos que recubren estas líneas de piedra (Fig. 6). La interpretación de estas formaciones es controvertida y hasta el momento no contamos con datos paleoambientales que confirmen los procesos climáticos que intervinieron en estas formaciones o en su defecto los procesos implicados en su formación.

Los yacimientos y hallazgos atribuidos a estos complejos se localizan en todo Africa Central: Gabón (Pomeret, 1965; Farine, 1963; Bayle des Hermens, et al, 1987; Locko, 1991; Clist, 1993; 1995); Camerún (Omi, 1977); República Popular del Congo (Lanfranchi, 1987; 1990; 1991a; Lanfranchi y Schwarz, 1990); República Democrática del Congo (Alimen, 1957; Van Moorsel, 1968; Cahen; 1978; 1982; Cahen y Moeyersons, 196971; Fiedler y Preuss, 1985; Preuss, 1990) y República Centroafricana (Bayle des Hermens, 1975). A pesar del número elevado de publicaciones sobre hallazgos atribuidos a estos complejos, los datos cronológicos, socioeconómicos y paleoclimáticos para esta parte del Continente siguen siendo practicamente inexistentes.

Durante la fase climática Kibanguiense, caracterizada por la introducción gradual del clima actual y el restablecimiento del bosque tal y como lo conocemos en la actualidad, se desarrollan una serie de industrias que 


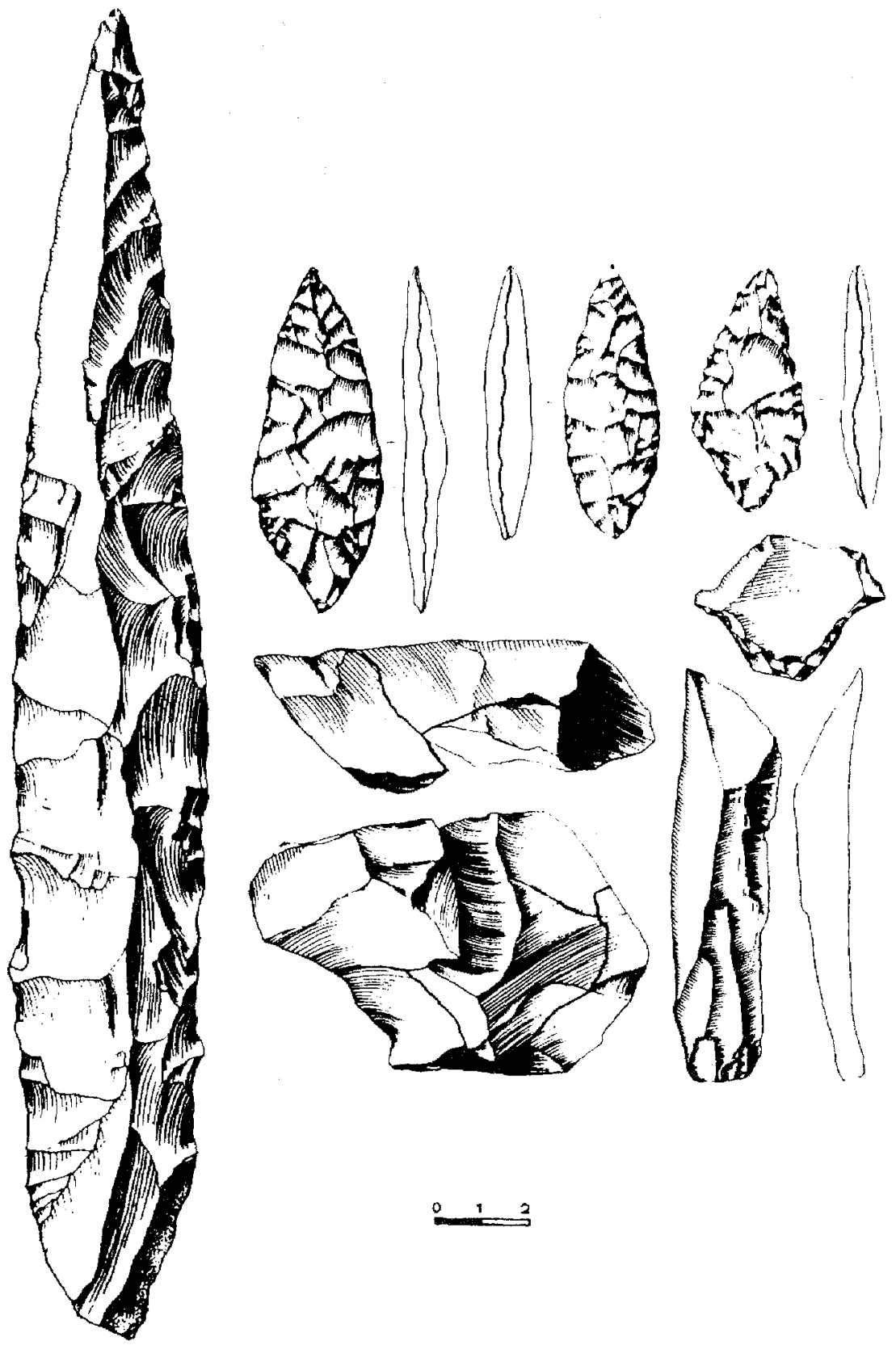

Fig. 5. Industria Lupembiense de Pointe de la Gombe (R.D.Congo) (Cahen, 1978). 

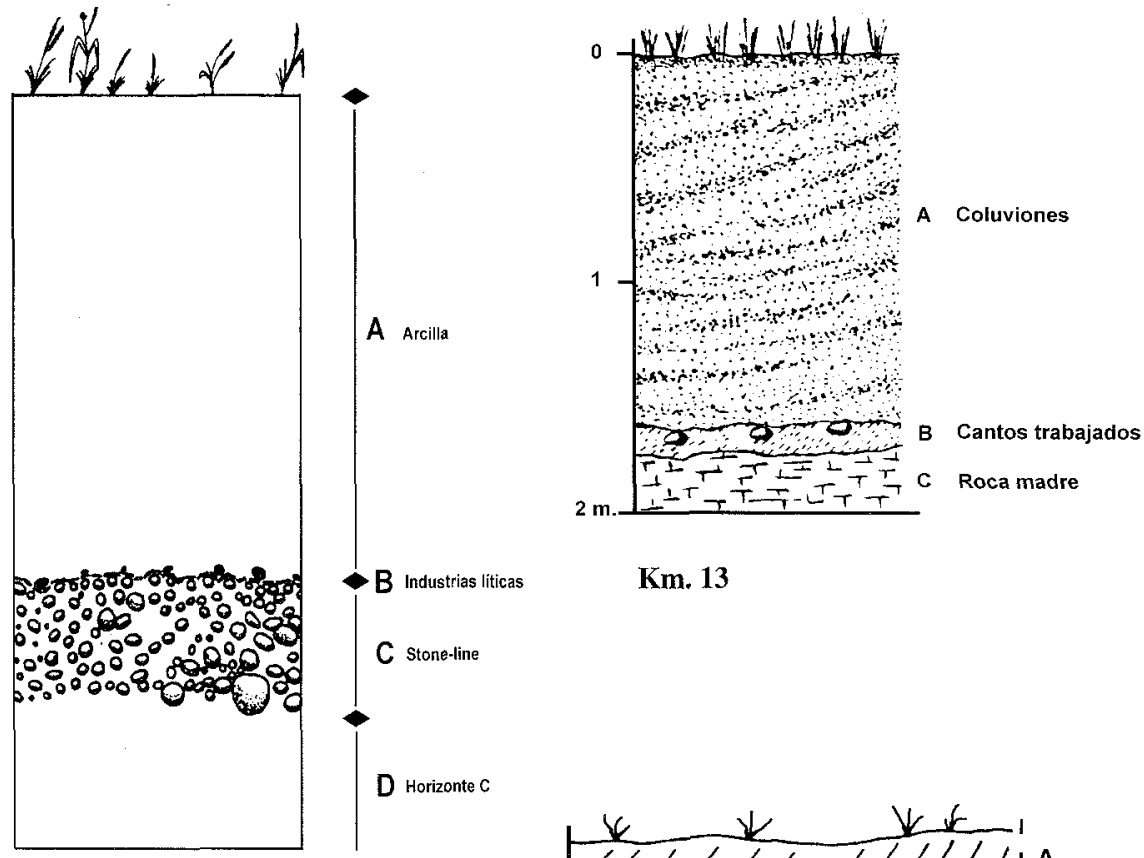

B Industrias liticas

Km. 13

C Stone-line

D Horizonte C

Kango

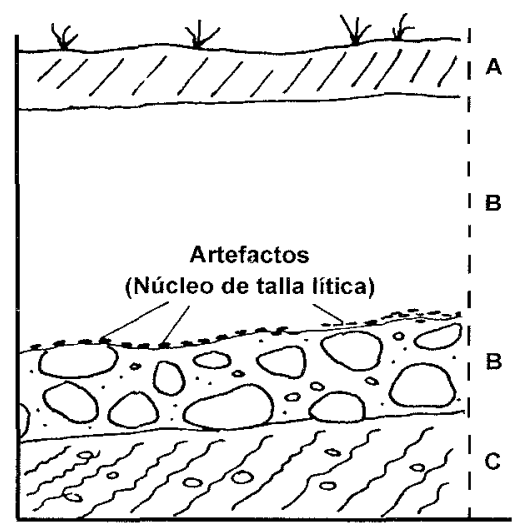

Mokeko

Fig. 6. Yacimientos arqueológicos en Stone-line: Kango (Gabón) (Clist, 1989a). Km. 13 (R.P.Congo) (Lanfranchi, 1987); Mokeko (R.P. Congo) (Lanfranchi y Schwartz, 1990). 
han sido englobadas bajo el término de Late Stone Age (LSA). La información obtenida en varios yacimientos de este periodo, permite acercarnos tímidamente a otros aspectos de la cultura difíciles de discernir en cronologías más antiguas. La mayor parte de las industrias se sitúan en el Holoceno antiguo y reciente, mientras que las fechas más antiguas se adentran en el Pleistoceno Final, siendo por lo tanto, contemporáneas con los yacimientos tardíos de la Middle Stone Age.

Dentro de la LSA, algunos complejos industriales como el Tshitoliense han sido diferenciados por presentar características tecno-tipológicas propias. Cronológicamente presenta un límite inferior de $14.840 \pm 80 \mathrm{BP}$ (GrN 7276) en las industrias Lupembo-tshitoliense de Pointe de la Gombe (Cahen, 1976) y un límite superior de $3.700 \pm 90$ BP (Gif 6504) en Louna, República Popular del Congo (Lanfranchi y Manima, 1989). Dataciones similares aparecen a lo largo de todo Africa Central (Van Moorsel, 1968; Emphoux, 1970; Cahen, 1976; Bayle des Hermens y Lanfranchi, 1978; Bayle des Hermens, 1981; Pincon, 1991).

Las industrias Tshitolienses (Fig. 7) se caracterizan por presentar elevados porcentajes de restos de talla (más del $90 \%$ ) y un número muy reducido de útiles que constituyen una quincena de tipos bien definidos (Lanfranchi, 1991b). Las piezas bifaciales en forma de "noyau de mangue», son particulares del Tshitoliense en esta parte de Africa Central y se reconocen por presentar un extremo distal pulido. Además de estos, son característicos los raspadores nucleiformes, cantos trabajados, raspadores espesos, raederas, cuchillos de dorso natural o abatido, muescas, denticulados, truncaturas, lascas, láminas retocadas o utilizadas. En cuanto a las puntas, las formas foliáceas, pedunculadas, de tranchet transversal y puntas con muesca, están también representadas. Los geométricos están presentes en la forma de segmentos y trapecios. El retoque suele ser por percusión, mientras que en las puntas, el sílex es calentado previamente para aplicar posteriormente el retoque por presión (Bayle des Hermens, 1981). Los núcleos más abundantes son los discoides planos, aunque también existen casos de talla bipolar y levallois. La materia prima utilizada es el silex, la cuarcita y eventualmente el cuarzo.

En términos generales el Tshitoliense se ha caracterizado por la tendencia a la disminución del tamaño de los artefactos, por la aparición de útiles de aspecto microlítico y de microlitos geométricos y por la generalización de las puntas de flecha que parecen atestiguar el uso del arco en este periodo.

Estos yacimientos se sitúan hoy día en la periferia del bosque centroafricano, en zonas dominadas por paisajes abiertos de sabana. Sin em- 

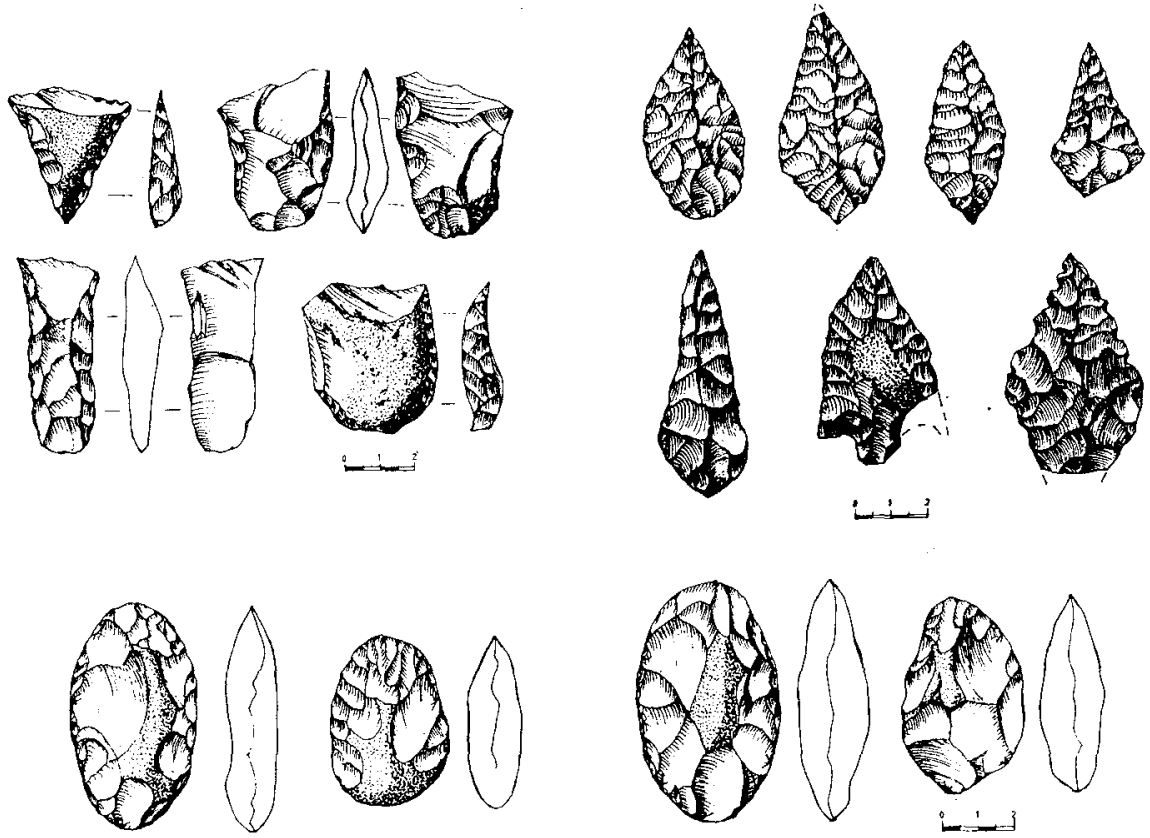

Fig. 7. Industrias Tshitolienses de la República Popular del Congo (Bayle des Hermens, 1981).

bargo, durante la fase climática en la que se desarrollan estas industrias, el Kibanguiense $A$, se produjo una expansión forestal más allá de sus límites actuales, por lo que estos enclaves en el momento de su ocupación se localizaban en contextos forestales y/o en el límite de estos. Es por ello que para algunos autores el Tshitoliense estaría caracterizado por la explotación de ecosistemas mixtos de sabana y bosque (Miller, 1988), lo cual podría verse avalado por esta localización sistemática en el curso inferior del río Congo y por la existencia en los yacimientos de restos faunísticos de especies de ambos ecosistemas (Van Neer y Lanfranchi, 1985).

El resto de las industrias de la LSA se caracterizan por el pequeño tamaño de sus artefactos, el predominio del cuarzo como soporte más común y el dominio de la percusión simple, así como algunos ejemplos de talla bipolar. Los restos de talla alcanzan porcentajes de más del $90 \%$. Entre los útiles destacan los raspadores, buriles, raederas, denticulados, perforadores, geométricos, hojas y hojitas. La talla levallois y los artefactos macrolíticos están presentes de forma eventual. 
Su distribución geográfica abarca la totalidad de Africa Central y presenta un marco cronológico mucho más amplio. En la periferia del bosque centroafricano ha sido datado en más de 40.700 años (GrN-7246) en Matupi Cave (Van Noten, 1977), mientras que en las tierras altas de Bamenda (Camerún) el yacimiento de Shum Laka presenta una primera fase de ocupación datada en $31.700 \pm 750$ BP (OxA-4945) (Cornelissen, 1996). En la zona forestal centroafricana se registra desde el Pleistoceno Final e inicios del Holoceno: $18.800 \pm 100 \mathrm{BP}$ (Os-21250) en Makubasi SE, en la República Democrática del Congo (Mercader, e.p.); $10.320 \pm$ 110 BP (Gif-A95561) en Gabón (Oslisly e.p.) y $6.020 \pm 505$ BP (Hv-10581) en Camerún (Maret, 1982a). Su límite inferior es controvertido dado que las ocupaciones de cazadores-recolectores con industrias líticas se mantienen avanzado el Holoceno reciente: $1.990 \pm 90$ BP (Gif-4391), en la República Democrática del Congo (Bayle des Hermens y Lanfranchi, 1978); $2.550 \pm 120$ BP (Beta-18729), en Gabón (Locko, 1988); y $2.680 \pm$ 50 BP (Gif-8150), en la República Popular del Congo, (Pincon, 1991).

Evidencias de actividades económicas centradas en la caza de mamíferos de mediano y gran tamaño, así como la recolección de gasterópodos han sido documentadas en los abrigos de Shum Laka, Abeke y Mbi, (Maret, et al, 1987; Asombang, 1991) todos ellos localizados en las tierras altas de Bamenda (Camerún), sin embargo, en el interior del bosque no se han registrado hasta el momento yacimientos con restos faunísticos en ocupaciones exclusivas de la LSA. La recolección vegetal está también mínimamente documentada. Los restos macrobotánicos de Canarium schweinfurthii recuperados en los yacimientos de la Región del Ituri (República Democrática del Congo), sugieren que las prácticas de arboricultura se podrían remontar a este periodo (Mercader, 1997).

Convencionalmente, al término de la Edad de la Piedra se registran los primeros yacimientos Neolíticos. Esta asignación se basa en dos tipos diferentes de observaciones: una es tecnológica y se aplica en general a las industrias que añaden en sus conjuntos cerámica y piedra pulimentada, la otra es económica e incluye solamente sociedades productoras de alimentos con evidencias de formas de vida sedentaria en poblados, almacenaje y agricultura. La confusión se crea cuando se mezclan ambos términos y se presupone que una innovación tecnológica como la cerámica es una evidencia tangible de cambios económicos relacionados con la producción de alimentos.

En diferentes zonas de Africa la asignación Neolítica se ha basado en este tipo de observaciones tecnológicas. Sin embargo, tanto la cerámica como la piedra pulimentada se registra con anterioridad en yacimientos 
de la LSA del Sahara (Mclntosh y McIntosh, 1983; Roset, 1983) y del Africa subsahariana (Shaw, 1978-79; Rosenfeld, 1972; Andah y Anozie, 1980); Gabel, 1976; Stahl, 1985; Smith, 1975; McIntosh y Mclntosh, 1983; Nygaard y Talbot, 1984). Por otra parte, excepto la certeza de una temprana utilización de Elaeis guineensis, no existe ninguna evidencia de domesticación de plantas y animales en Africa Central (Eggert, 1993:302). Debido a estos factores, T. Shaw (1978-79) recomienda abandonar el término de Neolítico y algunos investigadores (Mclntosh y Mclntosh, 1983, 1986; Eggert, 1993; Stahl, 1994) han optado por denominar estos yacimientos bajo el término de Late Stone Age cerámica, los cuales pueden estar o no asociados a evidencia agrícola.

En la secuencia prehistórica tradicional de Africa Central, el Neolítico esta íntimamente relacionado con la migración de poblaciones de lengua bantú. La glotocronología permite situar estos primeros movimientos de población alrededor del $5.000 \mathrm{BP}$, sobre un núcleo proto-bantú ya formado (Ehret, 1984; Vansina, 1984). A partir de este núcleo de origen, situado en algún punto ente Nigeria y Camerún, estas poblaciones comienzan una larga migración a través de todo Africa oriental, central y austral. Los estudios lingüísticos indican además, que estas poblaciones proto-bantúes eran ya agricultores, cultivaban ñame, calabazas y palmera de aceite, poseían animales domésticos como cabras y perros, habitaban en poblados de relativa envergadura y mantenían una organización social de cierta importancia de tipo bigman.

Estas interpretaciones lingüísticas e históricas sobre el origen de las poblaciones bantúes, han influido decisivamente en la interpretación de los yacimientos arqueológicos del Holoceno reciente. Estos enclaves han sido vistos como los restos materiales de poblaciones agrícolas bantúes que se instalan por primera vez en el bosque centroafricano (Van Noten, 1982; Clist, 1986; Oslisly y Fontugne, 1993) y cuya presencia producirá la desaparición, retirada y/o absorción de los grupos cazadores-recolectores que habitaban el territorio.

Una vez aceptada esta interpretación, la presencia de un hábitat neolítico ha sido definido por la aparición de cerámica, útiles pulimentados, material de molienda, pulidores, endocarpos de palmera de aceite; una cierta extensión en superficie de restos materiales, así como indicios de estructuras que indiquen una ocupación más prolongada del espacio (Clist y Jézégou, 1991: 165-166). Sin embargo, en la práctica, la presencia de cualquiera de estos marcadores en el registro arqueológico es utiliza como prueba irrefutable de las prácticas de producción llevadas a cabo por población bantu. 
Convencionalmente el Neolítico es seguido por el inicio de la Edad del Hierro, caracterizado por la introducción de las técnicas de fundición de metales como consecuencia de la llegada de una segunda oleada de población bantu en los bosques de Africa Central (Maret, 1989) que produce a su vez la desaparición de las poblaciones «neolíticas" locales.

Los yacimientos de la Edad del Hierro han sido caracterizados por presentar: (i) evidencia de fundición de metal en la forma de hornos, escorias, objetos o fragmentos de hierro, toberas, arcillas quemadas y en algunas ocasiones ladrillos de los hornos; (ii) aparición de nuevas formas y decoraciones cerámicas; (iii) aumento considerable de estructuras en forma de fosa, algunas de ellas excavadas a partir de un nivel de ocupación y a veces asociadas a agujeros de poste; (iv) restos de endocarpos de palmera de aceite; (v) evidencia indirecta de prácticas de agricultura en la forma de hachas y azadas de metal. Al igual que para el «Neolítico», cualquiera de estos marcadores ha servido para definir los yacimientos como unos enclaves de poblaciones bantúes de la Edad del Hierro.

La evidencia arqueológica, sin embargo, parece sugerir unos patrones de cambio tecnológico mucho más complejos:

- Yacimientos con industrias líticas asignables a la Late Stone Age que incorporan cerámicas en diferentes cronologías se constatan en Gabón en el $4.810 \pm 80$ BP (Beta-20789) y $3.400 \pm 70$ BP (Beta-17061) en Denis I y II (Clist, 1989b); $4.870 \pm 90$ BP (Gif-5987) en Sablières (Peyrot y Oslisly, 1987); en la República Democrática del Congo en el $2.985 \pm 41 \mathrm{BP}(\mathrm{Hv}-6254)$ en Kwimba (Maret, 1990) y $1.080 \pm 41 \mathrm{BP}$ (UtcNr 5075) en Matangai Turu NW (Mercader, 1997); y por último en la República Popular del Congo en el $2.880 \pm 90$ BP (Tx-5956) en Tchissanga (Denbow, 1990a).

- Durante el 3.000 BP-2.000 BP; o incluso en cronologías más tempranas, los yacimientos con cerámica y material de molienda se generalizan en la casi totalidad de Africa Central (Maret, 1982; 1983;1986; 1992; Peyrot y Oslisly, 1987, Eggert, 1987; Clist, 1988; 1995; Pincon, 1991; Oslisly y Fontugne, 1993; Oslisly, e.p.; etc.), al mismo tiempo aparecen en estos asentamientos una serie de estructuras en forma de fosas con unas dimensiones entre 50 y 200 centímetros de diámetro y de 120 a 200 centímetros de profundidad, relienas de sedimentos, fragmentos cerámicos, piedra pulimentada y materiai de molienda, así como semillas carbonizadas de Elaeis guineensis y de Canarium schweinfurthii (Fig. 8)

- Yacimientos con cronologías antiguas de la Edad del Hierro se conocen en Oliga, Camerún en el $2.820 \pm 100 \mathrm{BP}$ (Beta-31410) (Essomba, 1989); en Otoumbi II, Gabón en el $2.610 \pm 70$ BP (Beta-14834) (Oslisly y 

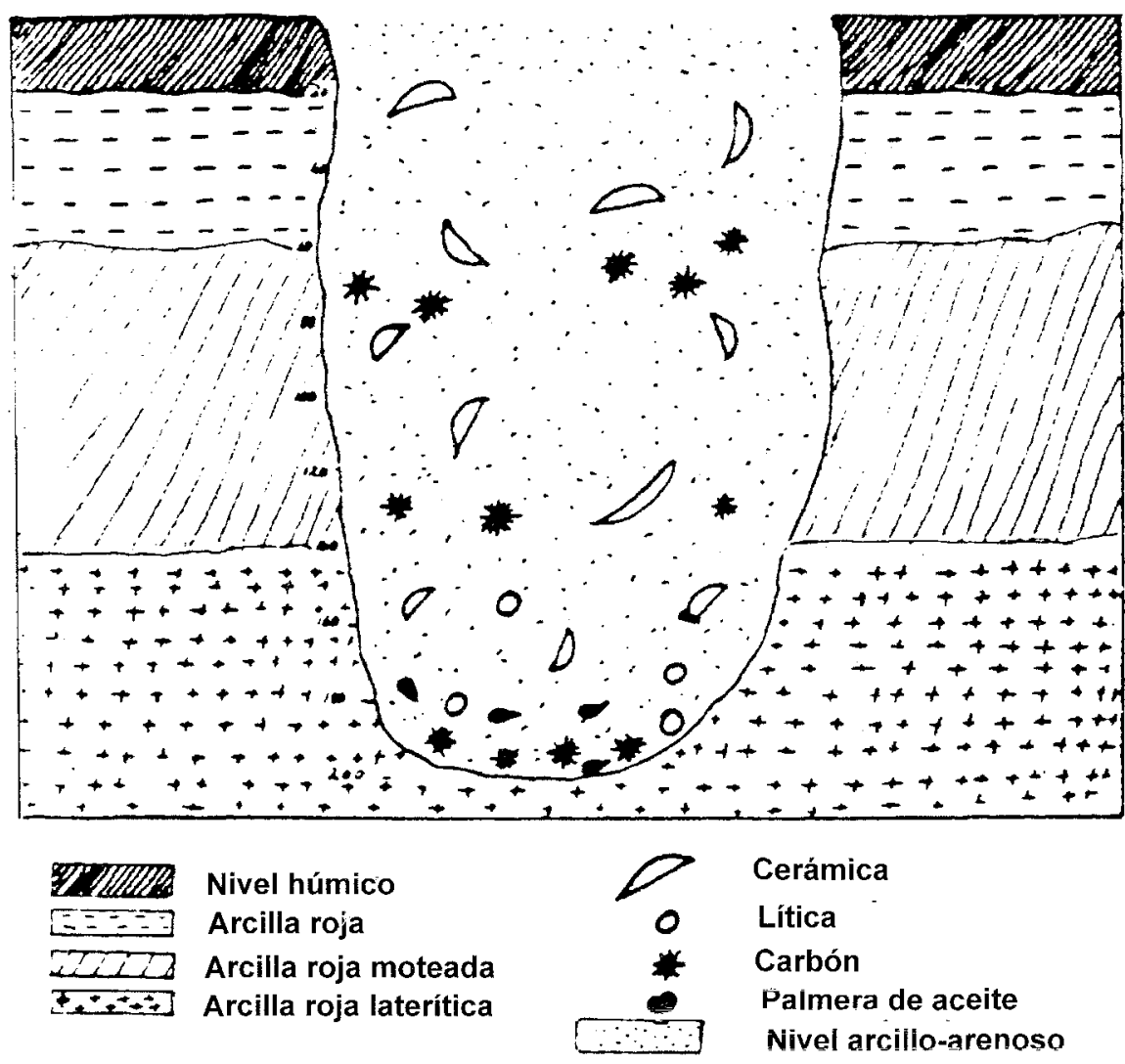

Cerámica

Lítica

Carbón

Palmera de aceite

Nivel arcillo-arenoso

Fig. 8. Fosa del Holoceno reciente, yacimiento de Nkometou (Camerún) (Essomba, 1992).

Fontugne, 1993); en Tchissanga Este, República Popular del Congo, en el $2.520 \pm 60 \mathrm{BP}$ (Tx6187) (Denbow, 1990a); y por último en Sazuki, República Democrática del Congo, 2.290 \pm 50 BP (Lv-1697) (Maret, 1990).

- Yacimientos con cerámicas en cronologías tardías sin evidencias de prácticas de fundición de metales se conocen en todo Africa Central: Tandou-Youmbi y Meningue (Denbow, 1990a; 1990b), Pointe Noire (Schwartz y Dechamps, 1991) y Case Barrier (Pincon, 1991), en la República del Congo y Kafele II (Clist, 1989a), Malekou (Locko, 1988), Ofoubou (Clist, 1992) en Gabón.

- Por último, ocupaciones de cazadores-recolectores con industrias líticas se mantienen avanzado el Holoceno reciente: $1.990 \pm 90$ BP (Gif- 
4391), en la República Democrática del Congo (Bayle des Hermens y Lanfranchi, 1978); $2.550 \pm 120$ BP (Beta-18729), en Gabón (Locko, 1988); y $2.680 \pm 50$ BP (Gif-8150), en la República Popular del Congo, (Pincon, 1991).

La mayor parte de estos yacimientos del Holoceno reciente son interpretados como resultado de la aparición en el bosque centroafricano de grupos de origen bantú, que con su presencia, desplazan a los grupos locales asentados con anterioridad en el territorio. La llegada de población, según esta interpretación, debería verse acompañada por cambios en los patrones de asentamiento, así como por la presencia de innovaciones tecnológicas. Por el contrario, los marcadores tecnológicos que definen el Neolítico, aparecen con anterioridad en asentamientos cazadores-recolectores locales. Además de ello, muchos de estos yacimientos presentan una ocupación anterior a la aparición de estas novedades y una presencia continuada a lo largo del tiempo en el que en algunos casos incorpora la nueva tecnología y en otros no.

Toda esta complejidad y variedad de asentamientos y tecnologías parecen indicar, más bien, la coexistencia de diferentes patrones de subsistencia durante un largo periodo de tiempo en Africa Central: (i) asentamientos cazadores-recolectores con diferentes grados de intensificación y diversificación económica; (ii) enclaves caracterizados por un descenso de la movilidad residencial, un incremento en el número de restos cerámicos, así como en endocarpos de palmera de aceite que podrían responder o no a asentamientos productores; y (iii) por último enclaves con las mismas características que los anteriores que incorporan la tecnología del hierro.

\section{CONCLUSIÓN}

El panorama arqueológico en el cinturón forestal centroafricano durante el Pleistoceno Final / Holoceno reciente es más complejo de lo que ha querido verse en un primer momento. La secuencia arqueológica africana ha sido construida a partir de paralelos con la Prehistoria europea (Edad de la Piedra / Neolítico / Edad del Hierro) y ha hecho de sus características la base de la interpretación de los yacimientos africanos. Sin embargo, la aplicación de esta secuencia no se ajusta con la complejidad y variabilidad del registro arqueológico centroafricano. Los patrones de cambio tecnológico presentan unas características propias que se desarrollan en algunas zonas en un momento muy temprano, mientras que en otras persisten hasta épocas tardías. Por otra parte, la interpretación de la aparición 
de novedades tecnológicas en periodos más recientes se ha basado en la aplicación de teorías migracionistas, en detrimento del desarrollo autóctono de las poblaciones locales. Un análisis más detallado de los periodos más recientes de la Prehistoria centroafricana, permite ver que ciertos eventos no encajan con la rigidez de la secuencia establecida, ni con la interpretación del origen alóctono de todas las novedades tecnológicas, económicas y culturales.

\section{BIBLIOGRAFÍA}

ADEBISI, M. (1991): Late Quaternary environments in Equatorial Africa: palynological evidence. Paleoecology of Africa, 22: 213-238.

Alimen, H. (1957): The Prehistory of Africa. Ed. Hutchinson. London.

ANDAH, B.W. y F. ANOzIE (1980): Preliminary report on the Prehistoric site of Afikpo (Nigeria). West African Journal of Archaeology, 10: 83-102.

Assombang, R. (1991): Cameroun. Age de la Pierre recent. In Lanfranchi, R. y B. Clist (eds): Aux origines de l'Afrique centrale: 99-101.

BAILEY, R.C. et al (1989): Hunting and gathering in tropical rainforest: is it possible? American Anthropologist, 91 (1): 59-82.

BAHUCHET, S. et al (1991): Wild yams revisited: Is independence form agriculture possible for rainforest hunter-gatherers? Human Ecology, 19 (2): 213-244.

Bayle Des Hermens, R. (1975): Recherches Préhistoriques en République Centrafricaine. Labethno, París.

Bayle Des Hermens, R. (1981): Note typologique sur le Tshitolien du Bassin du Congo. Prehistoire africaine: melangues offerts au doyen lionel Balout. ADPF. París.

Bayle Des Hermens, R. y R. Lanfranchi (1978): L'abri Tshitolien de Ntadi Yomba (République Populaire du Congo). L'Anthropologie, 82 (4): 539-564.

Bayle Des Hermens, R. R. Oslysli y B. Peyrot (1987): Premières séries de pierres taillés du paléolithique inférieur découvertes au Gabon, Afrique centrale. L'Anthropologie, 91 (2): 693-698.

BellWOOD, P. (1985): Prehistory of the Indo-malasyan archipielago. Academic Press. Sydney.

BISHOP, W.W. y J.D. CLARK (1967): Background to Evolution in Africa. Chicago: Univ. Chicago Press.

BouquiAuX, L. (1977): Actes du Colloque International du CNRS, Viviers (France), vol. 3 París, SELAF.

Cahen, D. (1976): Nouvelles fouilles a la Pointe de la Gombe (Ex-pointe de Kalina). Kinshasa, Zaïre. L'Antrhropologie, Tomo 80, n. ${ }^{\circ}$ 4: 573-602.

CAHEN, D. (1978): Vers une révision de la nomenclature des industries préhistoriques de L'Afrique centrale. L'Anthropologie. 82: n. 1: 5-36.

Cahen, D. (1982): The Stone Age in the South and West. En Van Noten, F. (ed): The Archaeology of Central Africa. Akademische druck-u. Verlagsanstalt Graz /Austria: 41-56.

CAHEN, D. y J. MoEYersons (1969-71): Le site prehistorique de la Kamoa, Katanga, Zaire. Palaeoecology of Africa, 6: 237-238.

CLARK, D. (1963): Prehistoric cultures of northeast Angola and their significance in tropical Africa. Companhia de Diamantes de Angola. Serviços Culturais. Museu do Dundo. Lisboa

CLARK, D. (1964): The Sangoan Culture of Equatoria: the implications of its stone equipment. Miscelanea en Homenage al Abate Henri Breuil. Institute de Prehistoria y Arqueología. Monografías IX. Barcelona: 309-325.

CLARK, D. (1982): The cultures of the Middle Palaeolithic / Middle Stone Age. In Clark, J.D. (ed.): Cambridge History of Africa, 1: 248-341.

CLARK, J.D. y COLE, S. (1957): The third Panafrican Congress on Prehistory, Livingstone, 1955. London: Chatto and Windus.

CLIST, B. (1986): Le Néolithique en Afrique Centrale: état de la question et perspective d'avenir. L'Anthropologie, 90 (2): 217-232. 
CLIST, B. (1988): Un nouvel ensemble néolithique en Afrique Centrale: le groupe d'Okala au Gabon. Nsi, 3: 43-51.

CLIST, B. (1989a): Bilan des premiers travaux du département d'archéologie du Ciciba: Missions du premier semestre 1985. En Obenga, T. (ed): Les Peuples bantu migrations, expansion et identite culturelle. L'Harmattan: 103-110.

CLIST, B. (1989b): Archaeology in Gabon, 1886-1988. The African Archaeological Review, 7: 5995.

CLIST, B. (1992):1991 excavations and laboratory work in Gabon. Nyame Akuma, 37: 4-8.

CLIST, B. (1993): Archaeological Fieldwork and Labwork in Gabon during 1992. Nyame Akuma, 39: $26-32$.

CLIST, B. (1995): Gabon 100.000 ans d'Histoire. Collection Découvertes du Gabon. Libreville: Centre Culturel Français. Saint Exupéry.

CLIST, B. y M.P. JEZEGOU (1991): Le néolithique: Gabon. En Lanfranchi, R. y B. Clist (eds): Aux origines de l'Afrique Centrale. CCF/CICIBA, París: 165-170.

ColinvauX, P.A. y M.B. Bush (1991): The rain forest ecosytem as a resource for hunting and gathering. American Anthropologist, 93 (1): 153-160.

ColinvauX, P.A. et al (1985): Discovery of permanent Amazon lakes and hydraulic disturbance in the upper Amazon basin. Nature, 313 (3): 42-45.

COON C.S; T. Shaw y S.G.H. DANIELS (1968): Yenguema cave report. University of Pennsylvania. Philadelphia.

CORNELISSEN, E. (1996): Shum Laka (Cameroon): late Pleistocene and early Holocene deposits. Aspect of African Archaeology. 257-263.

DAVIES, O. (1967): West Africa before the Europeans. London: Methuen.

DENBOW, J. (1990a): Congo to Kalahari: data and hypotheses about the political economy of the western stream of the Early Iron Age. The African Archaeological Review, 8: 139-176.

DENBOW, J. (1990b): Rapport preliminaire sur I'Archeologie du littoral congolais: prospections et fouilles de la region du Bas-kouilou effectuees en 1988. Nsi,7; 4-9.

EgGert, M.K.H. (1987): Imbonga and Batalimo: ceramic evidence for early settlement of the equatorial rain forest. The African Archaeological Review, 5: 129-145.

EgGeRT, M.K.H. (1993): Central Africa and the archaeology of the equatorial rainforest: reflections on some major topics. En Shaw, T. et al, (eds): The Archaeology of Africa, Food, metals and towns: 289-329.

EHRFT, C. (1984): Historical/linguistic evidence for early food production. En Clark, J.D. y S. Brandt (eds): From Hunters to farmers. The causes and consequences of food production in Africa: 26-35.

EmpHoux, J.P.(1970): La Grotte de Bitorri au Congo-Brazzaville. Cah. ORSTOM, Sér. Sci. Hum., VII (1): $3-20$.

EssombA, J.M. (1989): Dix ans de recherches archaeologiques au Cameroun Meridional. Nsi, 6: 33-57.

Essomba, J.M. (1992): Archéologie du Sud du Cameroun: notes préliminaires de recherches au site de Nkoumetou (Mfomakap). En Essomba, J.M. (ed): L'Archeologie au Cameroun. Actes du premie colloque international de Yaounde, 1986: 229-245.

FARINE, B. (1963): Sites préhistoriques gabonais, Ministère de l'Information au Gabon, Libreville.

Fernández, V.M., 1996: Arqueología Prehistórica de Africa. Editorial Síntesis. Madrid.

FIEDLER, L. y J. Preuss (1985): Stone tools form the Inner Zaïre Basin (Région de I'Equateur, Zaïre). The African Archaeological Review, 179-187.

FLENLEY, J.R. (1979): The equatorial rainforest: a geological history. Butterworths. Londres. 162 pág.

GABEL, C. (1976): Microlithic occurrences in the Republic of Liberia. West African Journal of Archaeology, 6: 21-35.

GENTRY, A.H. (1989): Speciation in tropical rainforest. En Holm-Nielsen, L.B., et al (eds)

Tropical forests. Botanical dynamics, speciation and diversity. 113-134.

HAFFER, J. (1969): Speciation in Amazonian forest Birds. Science, 165: 131-418.

HAMILTON, A.C. (1972-74): The significance of patterns of distribution shown by forest plants and animals in tropical Africa for the reconstruction of Upper Pleistocene paleoenvironments. Paleoecology of Africa, 9: 63-97.

HART, T.B. y J.A. HART (1986): The ecological basis of hunter-gatherer subsistence in African rain forest: the Mbuti of Eastern Zaire. Human Ecology, 14 (1): 29-55. 
HART, T.B., et al (1996): Changes in forest composition over the last 4.000 years in the Ituri basin, Zaire. En Van der Maesen, L.J.G., et al (eds): The biodiversity of African plants: 545-563.

HORSFALL, N. (1987): Living in rainforest: the prehistoric occupation of North Queensland's humid tropics. (Tesis doctoral inédita). Townsville. James Cook Univ.

LANFRANCHI, R. (1987): Nouvelles données sur la préhistoire de la Moyenne Vallée du Niari (République Populaire du Congo). Leba, 6: 5-22.

LANFRANCHI, R. (1990): Les industries préhistoriques en R.P. du Congo et leur contexto paléogéographique. En Lanfranchi, R. y D. Schwartz, (eds).: Paysages quaternaires du l'Africa centrale Atlantique: 406-417.

Lanfranchi, R. (1991a): Ages de la Pierre Ancien y Moyen. Congo. En Lanfranchi, R. y B. Clist (eds): Aux origines de l'Afrique centrale: 71-74.

Lanfranchi, R. (1991b): Age de la Pierre recent. Congo. En Lanfranchi, R. y B. Clist (eds): Aux origines de I'Afrique centrale: 111-114.

LANFRANCHI, R. y A. MANIMA (1989): Bilan de la recherche archeologique en R.P. du Congo, 1975-1989. Nsi, 6: 67-78.

LanfRanchi, R. Y SChWARTZ, D. (1990): Evolution des paysages de la Sangha (R.P. du Congo) au Pleistocène supérieur. Bilan des observations archéologiques, géomorphologiques, pédologiques et paléobiologiques. In LANFRANCHI, R. y CLIST, B. (Eds): Paysages Quaternaires de I'Afrique centrale Atlantique: 248-258.

LIVINGSTONE, D.A (1980): History of the tropical rainforest. Paleobiology, 6 (3): 243-244.

LOCKO, M. (1988): La recherche archéologique à I'Université Omar Bongo: Bilan scientifique. Muntu, 8: 26-44.

Locko, M. (1991): Ages de la Pierre Ancien y Moyen. Gabon. En Lanfranchi, R. y Clist, B. (Eds): Aux origines de l'Afrique centrale: 67-70.

MALEY, J. (1992): Mise en évidence d'une péjoration climatique entre ca. 2.500 et 2.000 ans b.p. en Afrique tropiale humide. Bull. Soc. Geolo. France, 1963 (3): 363-365.

MARET, P. (1982): Belgian archaeological project in Cameroon. Nyame Akuma, 20: 11-12.

Maret, P. (1983): Nouvelles donnees sur la fin de l'Age de la Pierre et les debuts de l'Age du Fer dans la moitie mericlionale du Cameroun. In Andah, B.; P. de Maret y R. Soper (eds): The proceedings of the 9th Congress of the Pan-African association of Prehistory and Related Studies. Jos 1983: 198-202.

MARET, P. (1984): I'Archéologie en zone bantu jusq en 1984. Muntu, 1 (2): 37-60.

MARET, P. (1985): Recent archaeological research and dates from Central Africa. Journal of African History, 26 (2-3): 129- 148.

MARET, P. (1986): The Ngovo Group: an industry with polished stone tools and poterry in Lower Zaïre. The African Archaeological Review, 4: 103-133.

MARET, P. (1989): Le contexte archéologique de l'expansion Bantu en Afrique centrale. Obenga, I.T. Les peuples bantu migrations, expansion et identite culturelle. L'Harmattan. Ciciba. Libreville: 118-138.

MARET, P. (1990): Le "Néolithique» et I'âge du fer ancien dans le sud-ouest de l'Afrique Centrale. En Lanfranchi, R. y D. Schwartz (eds): Paysages Quaternaires de I'Afrique Centrale Atlantique: 447-455.

MARET, P. (1992): Sédentarisation, agriculture et métallurgie du Sud-Cameroun. Synthèse des recherches depuis 1978. En Essomba, J.M. (de): L'Archéologie au Cameroun. Actes du premier Colloque International de Yaounde: 248-262.

Maret, P., B. Clist, W. Van Neer (1987): Résultats des premières fouilles dans les abris de Shum Laka et d'Abeke au Nord-Ouest du Cameroun. L'Anthropologie, 91, 2: 559-584.

McBreARTY, S. (1987): Une évaluation du Sangoen: son âge, son environnement et son rapport avec l'origine de l'Homo Sapiens. L'Anthropologie, $91, n .^{\circ} 2: 497-510$.

MCINTOSH, S.K. y R.J. McINTOSH (1983): Current Direction in West African Prehistory. Ann. Rev. Anthropology, 12: 215-258.

MCINTOSH, S.K. y R.J. MCINTOSH (1986): Recent Archaeological research and dates from West Africa. Journal of African History, 27: 413-442.

Mercader, J. (1997): Bajo el techo forestal. La evolución del poblamiento en el bosque ecuatorial del Ituri, Zaire. Tesis doctoral Inédita. Dpto. Prehistoria. Universidad Complutense de Madrid.

Mercader, J. (e.p.): Could prehistoric foragers live in rainforest? Later Stone Age in the tropical forest of Ituri, D.R. Congo. Current Anthropology. 
MERCADER, J., et al (en prensa): Late Pleistocene and Holocene environments in the northeast Congo Basin: archaeological perspectives form opal phytolith analysis.

MiLLER, S.I. (1988): Patterns of environment utilization by Late Prehistoric cultures in the Southern Congo Basin. En Bower, J. y D. Lubell (eds): Prehistoric cultures and environments in the Late Quaternary of Africa. Oxford: 127-144.

NYGAARD, S. y M.R. TALBOt (1984): Stone Age archaeology and environment on the southern Accra Plains, Ghana. Norwegian Archaeological Review, 17: 19-38.

Oliver, R. (1966): The problem of the Bantu expasion. Journal of African History, 7: 361-377.

OMI, G. (1977): Prehistoric sites and implements in Cameroon. An annex to the Interim Report of the Tropical African Geomorphology Research Project -1975/76. Nagoya. Japan.

Oslisly, R. (en prensa): The History of Human Settlement in the Middle Ogooué Valley (Gabon). Implications for the Environment. 26 pág.

Oslisly, R. y M. Fontugne (1993): La fin du stade néolithique et le début de l'âge du fer dans la moyenne vallée de I'Ogooué au Gabon. Problèmes chronologiques et changements culturels. C.R. Acad. Sci. Paris, 316, Serie II: 997-1003.

OSLISLY, R. y B. PEYrot (1988): Synthese des donnes archaeologiques des sites de la Moyenne Vallee de l'Ogooue (Province du Moyen Ogooué et Ogooué Ivindo) Gabon, Nsi, 3: 63-68.

Peyrot, B. y R. Oslisly (1987): Paleoenvironnement et archéologie au Gabon (1985-1986). Nsi, 1: 13-15.

PIncón, B. (1991): Archéologie des plateaux et collines Teke (R.P.Congo): de nouvelles donnes. Nsi, 8/9: 24-32.

POMMERET, I. (1965): Rapport de mission de recherches archéologiques dans le district de N’Djolé du 28 Décembre 1964 au 31 Décembre 1964. Bulletin de la Société Prehistorique et Protohistorique Gabonaise, 2: 27-29.

Preuss, J. (1990): L'évolution des paysages du bassin intérieur du Zaïre pendant les quarante derniers millénaires. En Lanfranchi, R. y D. Schwartz (eds): Paysages quaternaires de I'Afrique Centrale Atlantique: 260-270.

RANENE, A.J. (1972): Early human adaptations to New World tropical forest: a view form Panama. (Tesis doctoral inédita) University of California, Davis.

Roosevelt, A.C., et al (1996): Paleoindian cave dwellers in the Amazon: the peopling of the Americas. Science, 272: 378-384.

Rosenfeld, A. (1972): The microlithic Industries of Rop Rock shelter. West African Journal of Archaeology, 2: 17-18.

RoSET, J.P. (1983): Nouevelles données sur le probléme de la néolithisation du Sahara méridional: Air et Tenéré au Niger. Cahiers ORSTOM, ser. Géol, XIII (2): 119-142.

RUNGE, J. (1992): Geomorphological observations concerning palaeoenvironmental conditios in Eastern Zaire. Z. Geomorpho. NE, 91: 109-122.

SCHWARTZ, D. (1992): Asséchement climatiques vers 3000 b.p. et expansion Bantu en Afrique Centrale atlantique: quelques réflexions. Bull. Soc. Geol. France, 163 (3): 353-361.

SChWARTZ, D. y R. DEChamps (1991): Nouvelles ceramiques decouvertes a Pointe-Noire au Congo (1 $600 \mathrm{BP}$ ) au cours d'une fouille de sauvetage. Nsi, 8/9: 16-23.

SCHWARTZ, D. y R. LANFRANCHI (1993): Les cadres paléoenvironnementaux de I' évolution Humaine en Afrique Centrale Atlantique. L'Anthropologie, París (1), 97: 17-49.

SHAW, T. (1978-79): Holocene Adaptations in West Africa: The Late Stone Age. Early man news, 3-4: $51-82$.

Smith, A.B. (1975): Radiocarbon dates from Bosumpra Cave, Abetifi, Ghana. Proceeding of the Prehistoric Society, 41: 179-182.

STAHL, A.B. (1985): Reinvestigation of Kintampo 6 rock shelter, Ghana: implications for the nautre of culture chage. The African Archaeological Review, 3: 117-150.

STAHL, A.B. (1994): Innovation, diffusion and Culture Contact: The Holocene Archaeology of Ghana. Journal of World Prehistory, 8 (1): 51-112.

VAN Der Hammen T. (1991): Palaeoecological background: neotropics. Climatic change, 19 (1-2): $37-47$.

VAN NeER W. y R. LANFRANCHI (1985): Étude de la faune découverte dans l'abri Tschitolien de Ndati Yomba (République Populaire du Congo); L'Anthropologie, 89 (3): 351-364. 